\title{
Practice Spotlight: A Foundation for Mental Health in Children
}

\author{
Jay Barat, PharmD \\ Clinical Pharmacist, Mental Health and \\ Addictions Program \\ William Osler Health System - \\ Brampton Civic Hospital \\ Brampton, Ontario
}

$\mathrm{T}$ oo often, patients, families, and practitioners simply wait when faced with challenges related to mental health. This approach is particularly common when dealing with children presenting with symptoms that may indicate mental illness. Families wait and hope for improvement, practitioners wait for medications to take full effect before performing a reassessment, and patients wait for some resolution. As is the case for many illnesses, however, delays often result in escalation of the problem. For mental illness, such escalation means the complexity of psychosocial factors increases and the disease state continues to progress. As a consequence, the required services, medications, and overall management often become more complex and more challenging. Addressing complications as they present therefore becomes essential, especially in children and adolescents.

The William Osler Health System has a well-developed and comprehensive Mental Health and Addictions Program. Patients admitted into the Child and Adolescent (CHAD) mental health programs range in age from 5 to 17 years old. This time of life can be a challenging period for these vulnerable patients and their families. Initial diagnosis of one or more complications pertaining to mental health often occurs during this period. As patients and their families grapple with the disease state itself, they struggle to gain an overall understanding of both the disease and the therapeutic plan, while also facing significant life changes. The associated challenges include learning to navigate the health care system, attending frequent visits with physicians and counsellors, interacting with multiple health care providers, and understanding and coping with medication management. A pharmacist fits perfectly within the multidisciplinary team to address these concerns.

Optimizing medication regimens to maximize efficacy and limit adverse effects, developing a plan for success after discharge, and educating patients and their families about medications and expectations form the foundation for the contribution of clinical pharmacists to the CHAD mental health programs. Dr Jay Barat is one of these clinical pharmacists functioning with a unique scope of practice for this specialized population. He collaborates closely with a diverse multidisciplinary team that typically includes an occupational therapist, a dietitian, psychologists, social workers, child and youth counsellors, a recreational therapist, addiction counsellors, nurses, a pediatrician, and psychiatrists, in addition to the child and the family. For each patient, his goal is to provide an individualized, optimized therapeutic plan specifically as it pertains to medications.

Currently, Dr Barat provides group medication education to all patients admitted to the CHAD inpatient program. In more complex or high-risk cases, he provides individual counselling. Because of the heavy involvement of family members in the care and treatment of this vulnerable population, thorough counselling for the family is also provided. Over the past year, Dr Barat has counselled more than 500 children and adolescents and a large number of parents and other family members. During counselling sessions, the disease state, therapeutic plan, and expectations are all explained. Attention is given to describing the purpose of the medications, the reason for selecting the proposed regimen, the benefits and risks of the medications, and the role of pharmacotherapy in the overall therapeutic plan. These counselling efforts have resulted in positive outcomes for patients and families, as they are better equipped to achieve therapeutic goals. This benefit is reflected in the overwhelmingly positive feedback that is received from both patients and their families. In 2011, for example, 77 (91\%) of 85 inpatients surveyed reported that they had had a positive experience in the CHAD inpatient program. ${ }^{1}$ Among 55 parents surveyed, the average satisfaction rating was $90 \%$ for help from all staff members in the program. ${ }^{1}$ This positive feedback from patients and their families reflects the positive impact of the program as a whole. 
Dr Barat believes that a pharmacist's greatest contribution occurs in the setting of outpatient and community programs. Helping patients with mental health in the community can potentially address issues before they lead to hospital admissions. In addition, among patients who have been admitted to hospital, postdischarge work in the community will hopefully result in fewer readmissions.

In the near future, the William Osler Health System hopes to introduce a First Episode Schizophrenia Outpatient Program, with a focus on educating patients and families about this disease state, lifestyle management, and pharmacotherapy.
Although similar programs already exist in the region, none has a pharmacist as a core team member. It is Dr Barat's vision to expand the role of the pharmacist in programs such as this one to offer a strong foundation for the medication safety and wellbeing of patients with mental illness.

Reference

1. Krans TS. CHAD patient and parent satisfaction report. Brampton $(\mathrm{ON})$ : William Osler Health System, Mental Health and Addictions Program; 2011 Nov 10.

\title{
几 Canadian Society of Hospital Pharmacists Société canadienne des pharmaciens d'hôpitaux
}

\author{
Membership Year 2012/2013 (July 1, 2012 - June 30, 2013) \\ Are you a current member of the Canadian Society of Hospital Pharmacists?
}

CSHP has celebrated 65 years of inspiration and leadership among a rapidly growing network of over 3000 pharmacy colleagues. As a member of CSHP, you will have access to resources and opportunities that will enhance your career and help impact the direction of healthcare in Canada. Your participation in CSHP 2015, an initiative challenging all pharmacists to improve patient medication outcomes and safety through advancing pharmacy practice excellence by the year 2015 , is a prime example.

For more information about CSHP, please visit us at www.cshp.ca. We invite you to reap the benefits of an affordable membership with CSHP.

Membership Enquiries: Please contact Membership Services

Bus.: (613) 736-9733 Ext. 222 Fax: (613) 736-5660 Email: membershipservices@cshp.ca 